\title{
Pazopanib has equivalent anti-tumor effectiveness and lower Total costs than Sunitinib for treating metastatic or advanced renal cell carcinoma: a meta- analysis
}

Huan Deng ${ }^{1,2}$, Yu Huang ${ }^{2,3}$, Zhengdong Hong ${ }^{3}$, Xuhui Yuan ${ }^{2,3}$, Zhi Cao ${ }^{2,3}$, Yiping Wei ${ }^{1}$ and Wenxiong Zhang ${ }^{1^{*}}$ (D)

\begin{abstract}
Background: Sunitinib and pazopanib are extensively used as first-line treatment of metastatic renal cell carcinoma (mRCC). We performed this meta-analysis to assess the anti-tumor effectiveness, toxicity, and total costs of the two drugs among patients with mRCC/advanced RCC (aRCC).

Materials and Methods: PubMed, ScienceDirect, Scopus, Web of Science, Ovid MEDLINE, the Cochrane Library, Embase, and Google Scholar were searched to obtain eligible articles. The endpoints included progression-free survival (PFS), overall survival (OS), adverse effects (AEs), and per-patient-per-month (PPPM) costs.

Results: We included 14 medium- to high-quality studies. Both drugs were valid for mRCC/aRCC, with equivalent PFS (hazard ratio $(H R)=1.06,95 \%$ confidence interval [Cl]: 0.98-1.15, $P=0.13)$, OS (HR=0.92, 95\% Cl: 0.79-1.07, $P=0.29)$, objective response rate (ORR, risk ratio $(R R)=1.03,95 \% \mathrm{Cl}: 0.93-1.13, p=0.58)$, and disease control rate $(D C R, R R=1.03$, 95\% Cl: $0.94-1.22, P=0.54$ ). Sunitinib had more dosage reductions and higher PPPM (weighted mean difference $=-1.50$ thousand US dollars, $95 \% \mathrm{Cl}$ : -2.27 to $-0.72, P=0.0002$ ). Furthermore, more incidences of severe fatigue, thrombocytopenia, and neutropenia were recorded for sunitinib, but pazopanib had more liver toxicity. In subgroup analysis, studies from the US reported longer OS ( $\mathrm{HR}=0.86,95 \% \mathrm{Cl}: 0.77-0.95, P=0.004)$ and higher ORR $(\mathrm{RR}=1.24,95 \% \mathrm{Cl}: 1.03-1.51, P=0.03)$.

Conclusions: Pazopanib provides equivalent anti-tumor effectiveness and lower PPPM as compared with sunitinib for mRCC/aRCC. Great care should be given to pazopanib-treated patients with abnormal liver function. Nevertheless, more large-scale, high-quality studies are required.
\end{abstract}

Keywords: Meta-analysis, Pazopanib, Sunitinib, Targeted therapy, Renal cell carcinoma

\section{Background}

Renal cell carcinoma (RCC) is the eighth most common type of tumor, with 65,340 cases and an expected 14,970 deaths in 2018 [1]. Moreover, more than $30 \%$ of patients have metastases when initially diagnosed [2]. The US Food and Drug Administration has approved sunitinib

\footnotetext{
* Correspondence: zwx123dr@126.com

'Department of Thoracic Surgery, The First Affiliated Hospital of Nanchang University, Minde Rd, Nanchang 330006, Jiangxi Province, China Full list of author information is available at the end of the article
}

and pazopanib as first-line drugs for treating clear cell metastatic RCC (mRCC) [3, 4].

Sunitinib is an orally administered tyrosine kinase inhibitor (TKI) that has demonstrated efficacy and safety for mRCC in a randomized controlled trial (RCT) (NCT00130897) [5]. Pazopanib is also used as first-line TKI-targeted therapy for mRCC, and significantly promoted progression-free survival (PFS) and tumor response compared with placebo in patients with $\mathrm{mRCC} /$ advanced RCC (aRCC) in a randomized phase III clinical study [6]. Although both TKIs have shown superior

(c) The Author(s). 2019 Open Access This article is distributed under the terms of the Creative Commons Attribution 4.0 International License (http://creativecommons.org/licenses/by/4.0/), which permits unrestricted use, distribution, and reproduction in any medium, provided you give appropriate credit to the original author(s) and the source, provide a link to the Creative Commons license, and indicate if changes were made. The Creative Commons Public Domain Dedication waiver (http://creativecommons.org/publicdomain/zero/1.0/) applies to the data made available in this article, unless otherwise stated. 
benefits for treating $\mathrm{mRCC}$, the best patient profile for the two drugs is still unclear. In a phase III RCT, Motzer et al. reported that pazopanib had comparable PFS and overall survival (OS) to sunitinib, but the safety outcomes favored the pazopanib group [7]. However, a phase II RCT demonstrated no significant difference in the total number of adverse events (AEs) between the sunitinib and pazopanib groups [8]. Other studies indicated that sunitinib was associated with better OS than pazopanib [9].

To address this dispute, we performed a meta-analysis of relevant articles to compare the anti-tumor effectiveness, AEs, and per-patient-per-month (PPPM) costs of pazopanib and sunitinib to provide evidence-based suggestions for patients with $\mathrm{mRCC} / \mathrm{aRCC}$ in selecting first-line TKIs.

\section{Methods}

We performed this meta-analysis in accordance with preferred reporting items for systematic review and meta-analysis (PRISMA) guidelines (Additional file 1: Table S1).

\section{Search strategy}

PubMed, ScienceDirect, Scopus, Web of Science, Ovid MEDLINE, the Cochrane Library, Embase, and Google Scholar were searched up to September 2018 to select relevant articles comparing pazopanib versus sunitinib for $\mathrm{mRCC} / \mathrm{aRCC}$. The following terms were used: "pazopanib", "sunitinib", and "renal cell carcinoma". The integral search in PubMed was: (pazopanib [MeSH Terms] OR pazopanib [Text Word] OR GW786034B [Text Word] OR Votrient [Text Word]) AND (sunitinib [MeSH Terms] OR sunitinib [Text Word] OR sutent [Text Word] OR SU011248 [Text Word]) AND (renal cell carcinoma [MeSH Terms] OR renal cell carcinoma [Text Word]). We also searched the references of included studies to identify further eligible articles. All included articles were written in English.

\section{Inclusion criteria}

We included studies that met the following criteria: (1) patients were diagnosed with mRCC or aRCC (defined as regional lymph node metastasis and/or renal venous tumor thrombus and/or inferior vena cava tumor thrombus and/or adrenal metastasis or tumor infiltration with perirenal adipose tissue and/or renal sinus adipose tissue (but no more than the perirenal fascia), no distant metastasis of RCC); (2) pazopanib and sunitinib were compared; (3) results were PFS, OS, objective response rate (ORR), disease control rate (DCR), AEs, and PPPM; (4) written in English; (5) RCT or retrospective observational studies. We excluded reviews without raw data, meta-analysis, conference abstracts, case reports, and articles with repeated data.

\section{Data extraction}

Two investigators (Deng and Zhang) extracted the following information independently: first author, country, year of publication, the number of patients in two groups, study design, patient characteristics (age, sex, study period, research subjects, pre-treatment), anti-tumor effectiveness indicators (PFS, OS, ORR, DCR), total number of grade 3-4 AEs and PPPM. The data about the total health care costs of pazopanib and sunitinib were converted into PPPM through mathematical operations that total health care costs was divided by the number of patients and the duration of treatment (month). A third researcher (Hong) settled disagreements under various circumstances. We used hazard ratios (HRs), which consider the number and time of events, instead of odds ratios to analyze PFS and OS. HRs and 95\% confidence intervals (CIs) were obtained directly if Cox multivariate survival analysis was performed. Otherwise, HRs with 95\% CIs were extracted from Kaplan-Meier curves in accordance with Tierney et al. [10].

\section{Quality assessment}

We evaluated RCT quality using the 5-point Jadad scale, which includes questions on three major aspects: randomization, double-blinding, and withdrawals. A total score of $\geq 3$ points indicated that a study was high-quality [11].

We evaluated the quality of retrospective observational studies using the 9-point Newcastle-Ottawa Scale, which includes a questionnaire on three major aspects: selection, comparability, and exposure. A total score of 8-9 points indicated that a study was high-quality; a total score of 6-7 points indicated that a study was medium-quality [12].

\section{Statistical analysis}

We performed this meta-analysis using Review Manager (version 5.2) and STATA (version 12.0). HRs and 95\% CIs were used to analyze PFS and OS (HR $>1$ supports sunitinib group; $\mathrm{HR}<1$ supports pazopanib group). Risk ratios (RRs) and 95\% CIs were used to analyze the ORR, DCR ( $R R>1$ supports pazopanib group; $R R<1$ supports sunitinib group) and AEs ( $R R<1$ supports pazopanib group; RR $>1$ supports sunitinib group). Weighted mean differences (WMD) with 95\% CIs were used to analyze the PPPM. Subgroup analysis of PFS, OS, and ORR were performed to determine if these outcomes would vary according to country, the number of patients in pazopanib group, risk classification and study design. Heterogeneity was assessed using the $\chi^{2}$ test and $I^{2}$ statistic. If $I^{2}>50 \%$ or $P<0.1$, indicating significant heterogeneity, then the random-effects model would be used; if not, the fixed-effects model would be used. To enhance robustness, sensitivity analysis of PFS, OS, ORR, and 
DCR were performed to determine the effects of variables. We assessed publication bias through Begg's test and Egger's test. Additionally, if the bias caused by the combination of RCTs and retrospective studies was relatively small (for example, no significant difference was found in the subgroup analysis of PFS and OS about study design), we would analyze PFS and OS combining the two different types of studies. Otherwise, we would analyze RCTs and retrospective studies respectively. $P<0.05$ was considered a significant statistical difference.

\section{Results}

\section{Search results and quality evaluation}

Figure 1 shows the study selection process. An eventual 14 studies involving 12,985 patients (pazopanib, 3047; sunitinib, 9938) were selected for this meta-analysis [7-9, 13-23]. Three studies were RCTs (two studies stemmed from the same RCT with different aspects of results: one reported anti-tumor efficacy and toxicity; the other reported economic data. They were considered as multiple reports representing one RCT) and 10 were retrospective observational studies. Eight articles were high-quality (three RCTs scored $\geq 3$ points, one retrospective observational study scored 9 points, four retrospective observational studies scored 8 points). Five articles were medium-quality (four retrospective observational articles scored 7 points, one retrospective observational article scored 6 points). Table 1 lists the baseline characteristics and major evaluation indicators of all included articles.

\section{Anti-tumor effectiveness}

The anti-tumor effectiveness regarding PFS, OS, ORR, and DCR between the two groups was evaluated.

Three articles compared the PFS (heterogeneity: $\left.P=0.72, I^{2}=0 \%\right)$. There was no significant difference

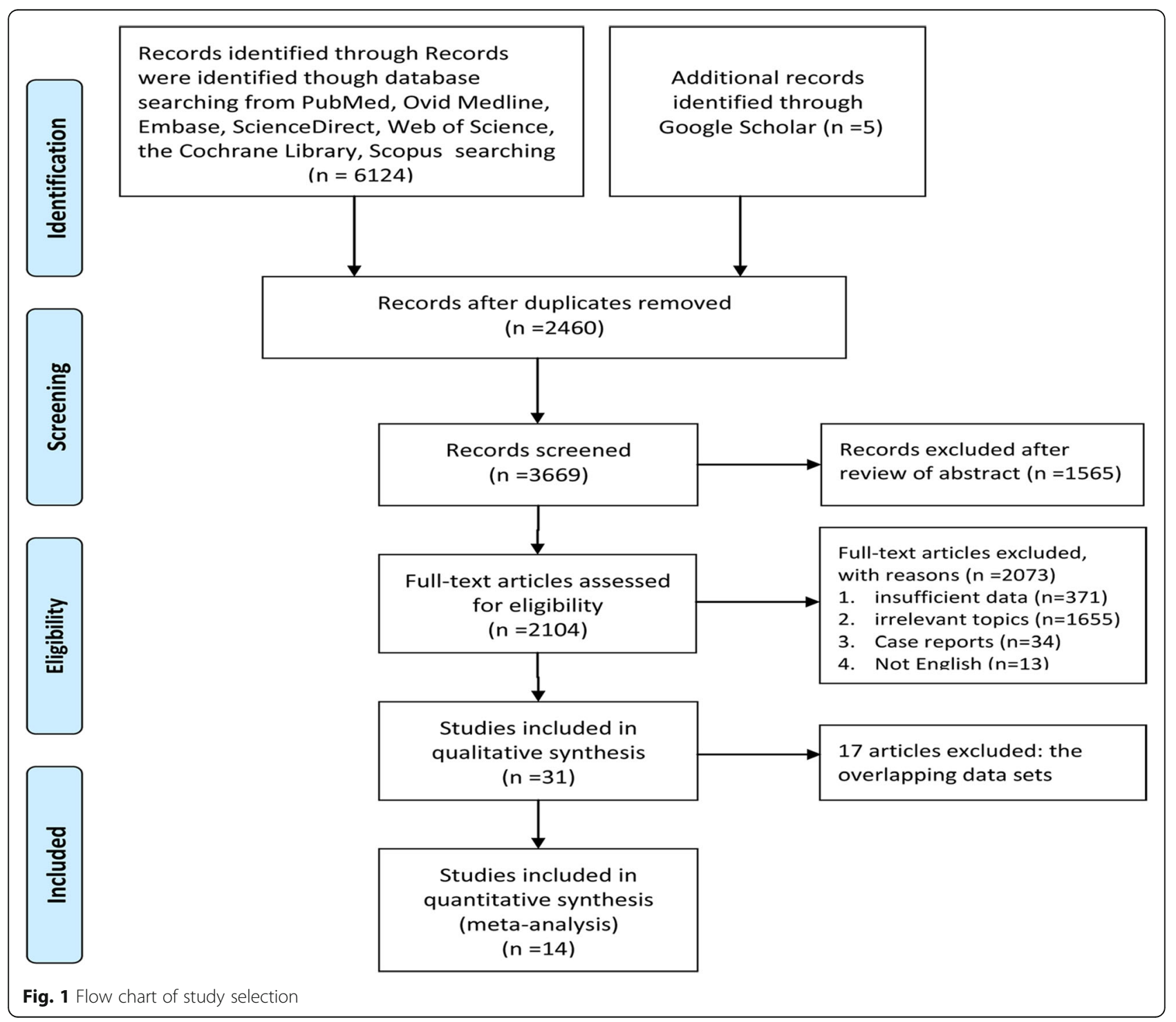




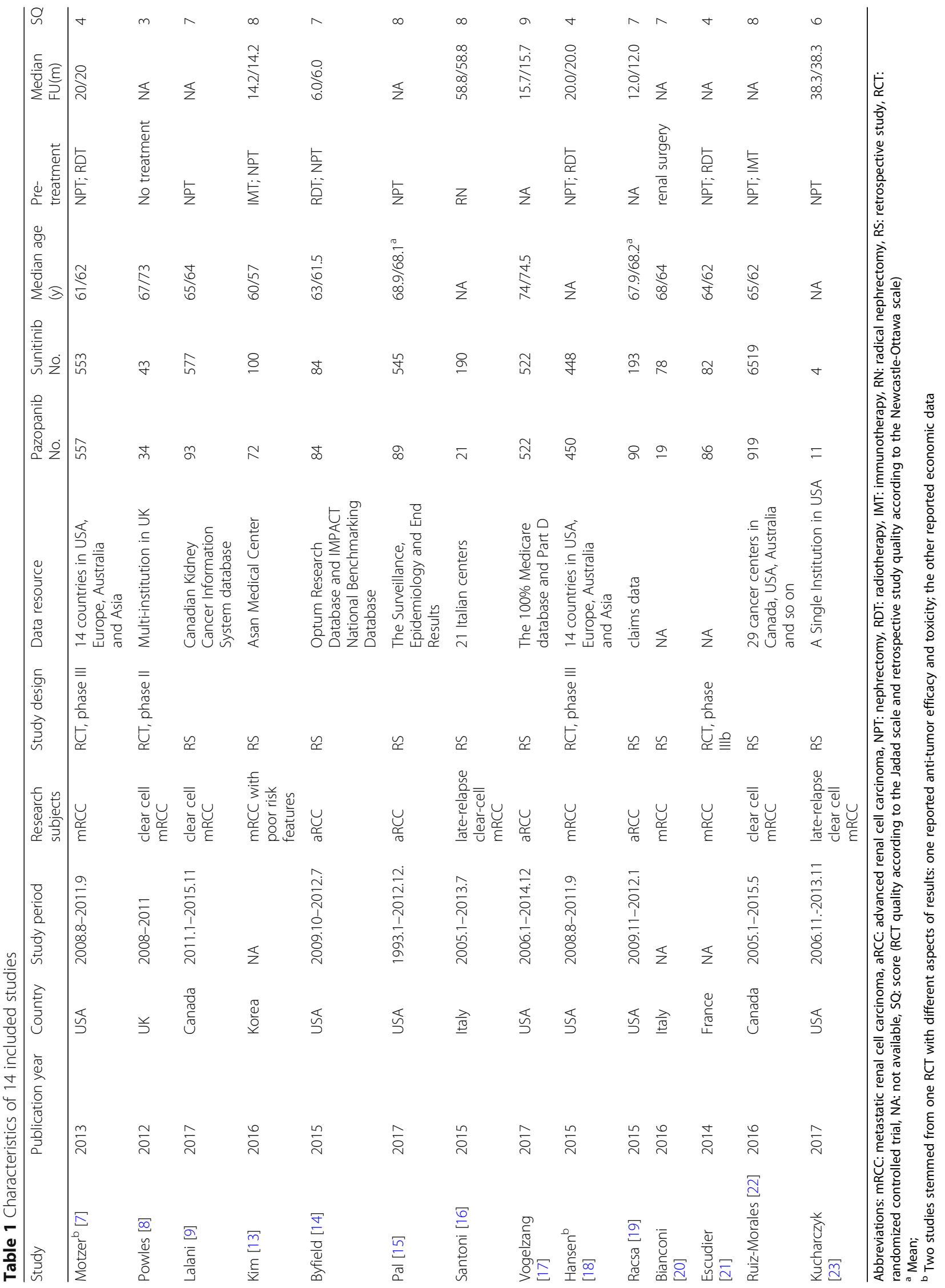




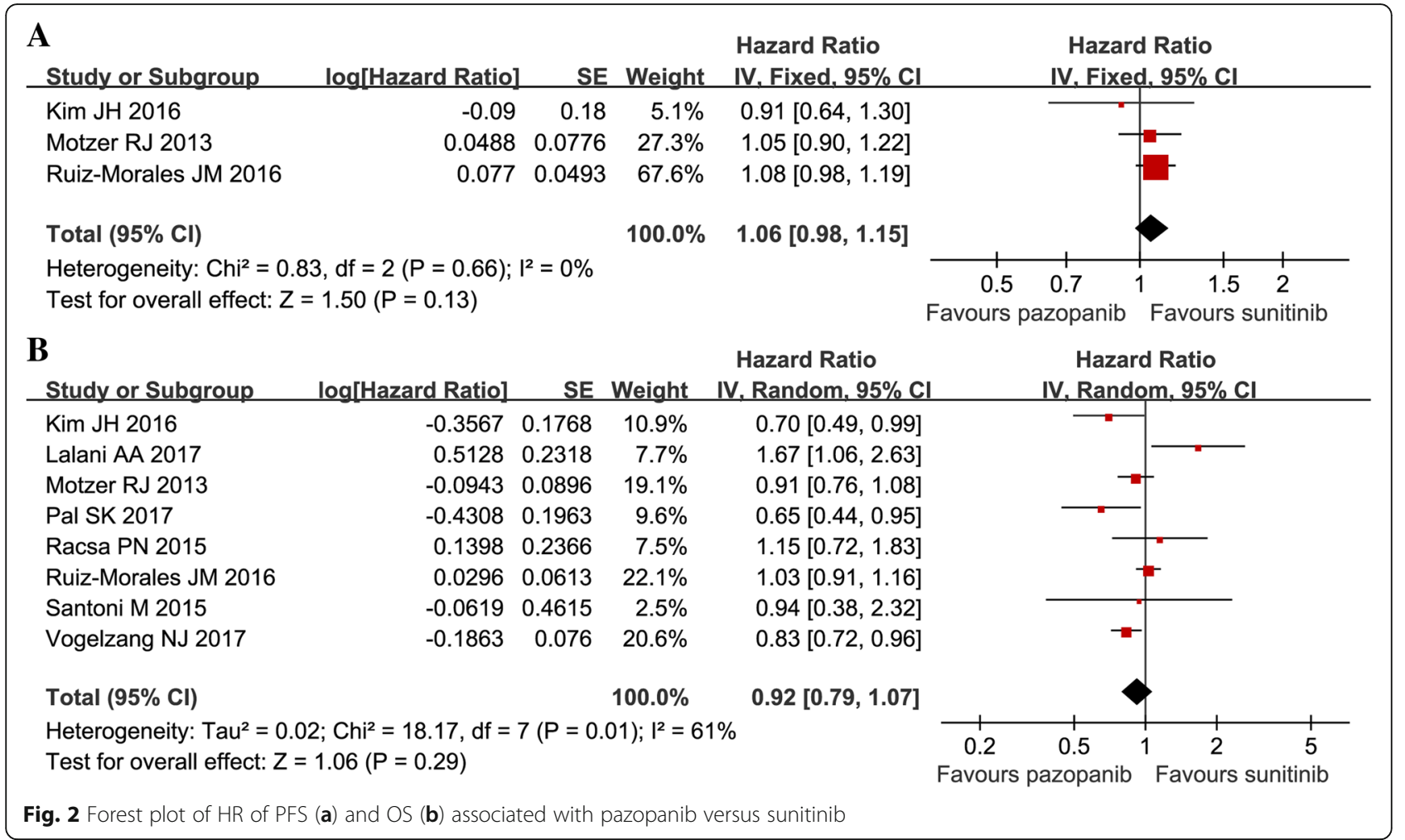

between pazopanib and sunitinib $(\mathrm{HR}=1.06,95 \% \mathrm{CI}$ : $0.98-1.15, P=0.13$; Fig. 2A).

Eight articles compared the OS (heterogeneity: $P=0.01$, $\left.I^{2}=61 \%\right)$. There was no significant difference between pazopanib and sunitinib (HR $=0.92,95 \% \mathrm{CI}$ : 0.79-1.07, $P=0.29$; Fig. 2B).

Eight articles compared the ORR (heterogeneity: $\left.P=0.06, I^{2}=48 \%\right)$. There was no significant difference between pazopanib and sunitinib $(\mathrm{RR}=1.03,95 \% \mathrm{CI}$ : 0.93-1.13, $P=0.58$; Fig. 3A).

Seven articles compared the DCR (heterogeneity: $\left.P=0.03, I^{2}=58 \%\right)$. There was no significant difference between pazopanib and sunitinib $(\mathrm{RR}=1.03,95 \% \mathrm{CI}$ : 0.94-1.22, $P=0.54$; Fig. 3B).

\section{Toxicity}

We compared grade 3-4 toxic events and performed subgroup analysis of the 10 most common toxic events.

Two articles compared grade 3-4 AEs (heterogeneity: $\left.P=0.04, I^{2}=76 \%\right)$. There was no significant difference between the two groups $(\mathrm{RR}=0.63,95 \% \mathrm{CI}$ : 0.19-2.12, $P=0.46$; Fig. 4A).

Some patients experienced drug discontinuation or reductions. Three studies compared drug discontinuations; there was no significant difference between the two groups $(\mathrm{RR}=0.96,95 \% \mathrm{CI}$ : 0.84-1.10, $P=0.57$; Fig. 4B). Three studies compared drug reductions; the sunitinib group had more drug reductions $(\mathrm{RR}=0.86,95 \% \mathrm{CI}$ :
0.76-0.97, $P=0.01$; Fig. 4C). Four studies compared drug discontinuations due to serious AEs; no significant difference was found $(\mathrm{RR}=1.16,95 \% \mathrm{CI}$ : 0.98-1.37, $P=0.08$; Fig. 4D).

In the subgroup analysis of the 10 most common AEs (diarrhea, fatigue, hypertension, nausea/vomiting, leukopenia, thrombocytopenia, neutropenia, increased creatinine, increased aspartate aminotransferase [AST], and increased alanine aminotransferase [ALT]), the outcomes of these grade 3-4 AEs indicated that there were no significant differences in hypertension, nausea/vomiting, and increased AST between the two groups. For all grade AEs, sunitinib had higher incidences of fatigue (RR $=0.87$, 95\% CI: 0.79-0.96, $P=0.006)$, leukopenia (RR = 0.55, 95\% CI: 0.50-0.61, $P<0.00001)$, thrombocytopenia $(\mathrm{RR}=0.54,95 \% \mathrm{CI}: 0.48-0.60, P<0.00001)$, neutropenia $(\mathrm{RR}=0.54,95 \% \mathrm{CI}: 0.48-0.61, P<0.00001)$, and increased creatinine $(\mathrm{RR}=0.68,95 \% \mathrm{CI}: 0.59-0.79, \quad P<0.00001)$; pazopanib induced significantly higher rates of diarrhea ( $\mathrm{RR}=1.11,95 \% \mathrm{CI}: 1.01-1.23, P=0.03)$ and increased ALT $(\mathrm{RR}=1.35$, 95\% CI: $1.20-1.51, P<0.00001$, Table 2$)$. The outcomes of these grade 3-4 AEs demonstrated no significant differences were found for diarrhea, hypertension, nausea/vomiting, leukopenia, and increased creatinine between pazopanib and sunitinib. For grade 3-4 AEs, sunitinib had more fatigue ( $R R=0.59,95 \% \mathrm{CI}$ : 0.44-0.80, $P=0.0006$ ), thrombocytopenia ( $R R=0.16,95 \%$ CI: $0.10-0.25, \mathrm{P}<0.00001)$, and neutropenia $(R R=0.23$, 


\begin{tabular}{|c|c|c|c|c|c|c|c|}
\hline $\begin{array}{l}\text { A } \\
\text { Study or Subgroup }\end{array}$ & $\begin{array}{l}\text { pazop } \\
\text { Events }\end{array}$ & $\begin{array}{l}\text { anib } \\
\text { Total }\end{array}$ & $\begin{array}{r}\text { suniti } \\
\text { Events }\end{array}$ & inib & al Weight & $\begin{array}{c}\text { Risk Ratio } \\
\text { M-H, Fixed, } 95 \% \mathrm{CI}\end{array}$ & $\begin{array}{c}\text { Risk Ratio } \\
\text { M-H, Fixed, } 95 \% \mathrm{Cl}\end{array}$ \\
\hline Bianconi M 2016 & 2 & 19 & 10 & 7 & $0.7 \%$ & $0.82[0.20,3.44]$ & \\
\hline Escudier B 2014 & 16 & 85 & 17 & 8 & $3.0 \%$ & $0.89[0.48,1.63]$ & \\
\hline Kim JH 2016 & 26 & 72 & 23 & 10 & $3.3 \%$ & $1.57[0.98,2.52]$ & \\
\hline Kucharczyk J 2017 & 2 & 11 & 1 & & $0.1 \%$ & $2.08[0.12,36.07]$ & \\
\hline Motzer RJ 2013 & 171 & 557 & 137 & 55 & $23.6 \%$ & $1.24[1.02,1.50]$ & $=$ \\
\hline Powles T 2012 & 5 & 34 & 6 & 4 & $0.9 \%$ & $1.05[0.35,3.16]$ & \\
\hline Ruiz-Morales JM 2016 & 196 & 707 & 1687 & 556 & $65.3 \%$ & $0.91[0.81,1.04]$ & \\
\hline Santoni M 2015 & 14 & 21 & 92 & 19 & $3.1 \%$ & $1.38[0.98,1.93]$ & \\
\hline Total $(95 \% \mathrm{Cl})$ & & 1506 & & 660 & $9 \quad 100.0 \%$ & $1.03[0.93,1.13]$ & 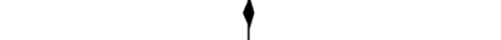 \\
\hline Total events & 432 & & 1972 & & & & \\
\hline $\begin{array}{l}\text { Heterogeneity: } \text { Chi }^{2}=1 \\
\text { Test for overall effect: } Z\end{array}$ & $\begin{array}{l}3.59, \mathrm{df}=7 \\
=0.56(P\end{array}$ & $\begin{array}{l}7(P=0 \\
=0.58)\end{array}$ & $0.06) ; 1^{2}=$ & $48 \%$ & & & 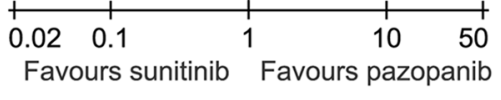 \\
\hline $\begin{array}{l}\text { B } \\
\text { Study or Subgroup }\end{array}$ & $\begin{array}{l}\text { pazopan } \\
\text { Events }\end{array}$ & $\begin{array}{l}\text { nib } \\
\text { Total }\end{array}$ & $\begin{array}{l}\text { sunitini } \\
\text { Events }\end{array}$ & $\begin{array}{l}\text { ib } \\
\text { Total }\end{array}$ & Weight & $\begin{array}{c}\text { Risk Ratio } \\
\text { M-H, Random, } 95 \% \mathrm{Cl}\end{array}$ & $\begin{array}{c}\text { Risk Ratio } \\
\mathrm{M}-\mathrm{H}, \text { Random, } 95 \% \mathrm{Cl}\end{array}$ \\
\hline Bianconi M 2016 & 9 & 19 & 33 & 78 & $2.4 \%$ & $1.12[0.65,1.92]$ & \\
\hline Escudier B 2014 & 48 & 85 & 55 & 80 & $9.6 \%$ & $0.82[0.65,1.04]$ & 7 \\
\hline Kim JH 2016 & 59 & 72 & 60 & 100 & $12.7 \%$ & $1.37[1.13,1.66]$ & $\mp$ \\
\hline Kucharczyk J 2017 & 10 & 11 & 2 & 4 & $0.7 \%$ & $1.82[0.67,4.93]$ & \\
\hline Motzer RJ 2013 & 387 & 557 & 379 & 544 & $26.7 \%$ & $1.00[0.92,1.08]$ & 1 \\
\hline Powles T 2012 & 34 & 34 & 43 & 43 & $30.4 \%$ & $1.00[0.95,1.05]$ & 1 \\
\hline Santoni M 2015 & 19 & 21 & 172 & 190 & $17.4 \%$ & $1.00[0.86,1.16]$ & + \\
\hline Total $(95 \% \mathrm{Cl})$ & & 799 & & 1039 & $100.0 \%$ & $1.03[0.94,1.12]$ & 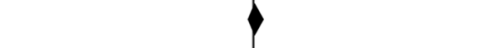 \\
\hline Total events & 566 & & 744 & & & & \\
\hline \multicolumn{7}{|c|}{$\begin{array}{l}\text { Heterogeneity: } \mathrm{Tau}^{2}=0.01 ; \mathrm{Chi}^{2}=14.28, \mathrm{df}=6(P=0.03) ; \mathrm{I}^{2}=58 \% \\
\text { Test for overall effect: } Z=0.61(P=0.54)\end{array}$} & 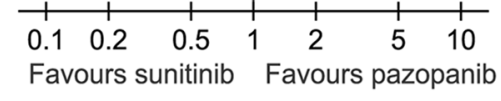 \\
\hline
\end{tabular}

95\% CI: $0.15-0.34, \mathrm{P}<0.00001)$, but pazopanib had significantly higher incidences of increased AST (RR = 4.46, 95\% CI: 2.62-7.58, P $<0.00001)$ and increased ALT $(\mathrm{RR}=4.34,95 \% \mathrm{CI}:$ 2.79-6.75, $\mathrm{P}<0.00001$; Table 3$)$.

\section{PPPM}

We assessed total costs between the pazopanib and sunitinib groups based on the PPPM. Two studies compared PPPM (heterogeneity: $P=0.81, I^{2}=0 \%$ ); the pazopanib group had significantly lower PPPM (WMD $=-1.50$ thousand US dollars, $95 \% \mathrm{CI}:-2.27$ to $-0.72, P=0.0002$; Fig. 5).

\section{Subgroup analysis}

To determine if the anti-tumor effectiveness of pazopanib vs. sunitinib would be consistent across subgroups, the pooled results for PFS, OS, and ORR were calculated according to country, the number of patients in pazopanib group, risk classification, and study design (Table 4). Interestingly, the pooled results of included studies from the US found pazopanib had longer OS $(\mathrm{HR}=0.86,95 \%$ CI: $0.77-0.95, P=0.004)$ and higher ORR ( $R R=1.24$, 95\% CI: $1.03-1.51, P=0.03)$, the pooled results of included studies from Korea also found pazopanib had improved OS ( $\mathrm{HR}=0.70,95 \% \mathrm{CI}: 0.49-0.99, P=0.04)$, and the pooled results of RCT found pazopanib had better ORR $(\mathrm{HR}=1.19,95 \% \mathrm{CI}: 1.00-1.43, \quad P=0.05)$ although the difference wasn't significant.

\section{Sensitivity analysis}

PFS (Additional file 2: Figure S1A), OS (Additional file 2: Figure S1B), and DCR (Additional file 3: Figure S2B) were all robust: sensitivity analysis showed consistent results. However, the sensitivity analysis of ORR (Additional file 3: Figure S2A) showed that the estimate of the study Ruiz-Morales et al. [18] exceeded the 95\% CI.

\section{Publication Bias}

There was no proof of publication bias in PFS (Begg's test, $p=0.296$, Egger's test, $P=0.058$; Additional file 4 : Figure S3A), OS (Begg's test, $P=0.902$; Egger's test, $P=0.951$; Additional file 4: Figure S3B), ORR (Begg's test, $P=0.536$; Egger's test, $P=0.904$; Additional file 5: Figure S4A), and DCR (Begg's test, $P=0.806$; Egger's test, $P=0.479$; Additional file 5: Figure $\mathrm{S} 4 \mathrm{~B}$ ). 


\begin{tabular}{|c|c|c|c|c|c|c|c|}
\hline $\begin{array}{l}\text { A } \\
\text { Study or Subgroup }\end{array}$ & $\begin{array}{l}\text { pazopan } \\
\text { Events }\end{array}$ & $\begin{array}{l}\text { iib } \\
\text { Total } \\
\end{array}$ & $\begin{array}{l}\text { sunitini } \\
\text { Events }\end{array}$ & $\begin{array}{l}\text { ib } \\
\text { Total } V\end{array}$ & Weight & $\begin{array}{c}\text { Risk Ratio } \\
\text { M-H, Random. } 95 \% \mathrm{Cl}\end{array}$ & $\begin{array}{c}\text { Risk Ratio } \\
\mathrm{M}-\mathrm{H}, \text { Random, } 95 \% \mathrm{Cl}\end{array}$ \\
\hline Motzer RJ 2013 & 412 & 554 & 402 & 548 & $61.8 \%$ & $1.01[0.94,1.09]$ & \\
\hline Powles T 2012 & 3 & 67 & 13 & 85 & $38.2 \%$ & $0.29[0.09,0.99]$ & \\
\hline Total $(95 \% \mathrm{Cl})$ & & 621 & & 6331 & $100.0 \%$ & $0.63[0.19,2.12]$ & \\
\hline Total events & 415 & & 415 & & & & \\
\hline $\begin{array}{l}\text { Heterogeneity: } \mathrm{Tau}^{2}= \\
\text { Test for overall effect: }\end{array}$ & $\begin{array}{l}.62 ; \mathrm{Chi}^{2}= \\
=0.75(\mathrm{P}\end{array}$ & $\begin{array}{l}=4.20 \\
=0.46\end{array}$ & $d f=1(P=$ & $=0.04)$; & $;\left.\right|^{2}=76 \%$ & & $\begin{array}{ccccc}0.02 & 0.1 & 1 & 10 & 50 \\
\text { Favours pazopanib } & \text { Favours sunitinib }\end{array}$ \\
\hline $\begin{array}{l}\text { B } \\
\text { Study or Subgroup }\end{array}$ & $\begin{array}{l}\text { pazopa } \\
\text { Events }\end{array}$ & $\begin{array}{l}\text { Inib } \\
\text { Total }\end{array}$ & $\begin{array}{l}\text { sunitir } \\
\text { Events }\end{array}$ & $\begin{array}{l}\text { nib } \\
\text { Total }\end{array}$ & Weight & $\begin{array}{l}\text { Risk Ratio } \\
\text { M-H, Fixed, } 95 \% \mathrm{Cl}\end{array}$ & $\begin{array}{l}\text { Risk Ratio } \\
\text { M-H, Fixed, } 95 \% \mathrm{Cl}\end{array}$ \\
\hline Byfield SA 2015 & 39 & 84 & 37 & 84 & $28.0 \%$ & $1.05[0.76,1.47]$ & \\
\hline Kim JH 2016 & 63 & 72 & 98 & 100 & $62.0 \%$ & $0.89[0.81,0.98]$ & \\
\hline Racsa PN 2015 & 11 & 57 & 21 & 123 & $10.0 \%$ & $1.13[0.59,2.18]$ & \\
\hline Total $(95 \% \mathrm{Cl})$ & & 213 & & 307 & $100.0 \%$ & $0.96[0.84,1.10]$ & \\
\hline Total events & 113 & & 156 & & & & \\
\hline $\begin{array}{l}\text { Heterogeneity: } \mathrm{Chi}^{2}= \\
\text { Test for overall effect: }\end{array}$ & $\begin{array}{l}3.05, \mathrm{df}=2 \\
Z=0.57(F\end{array}$ & $\begin{array}{l}2(P=0 \\
P=0.5\end{array}$ & $\begin{array}{l}0.22) ; 1^{2}= \\
77)\end{array}$ & $34 \%$ & & & 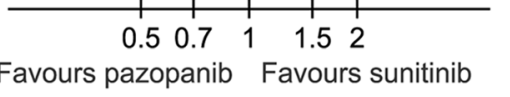 \\
\hline $\begin{array}{l}\text { C } \\
\text { Study or Subgroup }\end{array}$ & $\begin{array}{l}\text { pazopa } \\
\text { Events }\end{array}$ & $\begin{array}{l}\text { Inib } \\
\text { Total }\end{array}$ & $\begin{array}{l}\text { sunitir } \\
\text { Events }\end{array}$ & $\begin{array}{l}\text { nib } \\
\text { Total } \\
\end{array}$ & Weight & $\begin{array}{l}\text { Risk Ratio } \\
\text { M-H. Fixed, } 95 \% \mathrm{Cl}\end{array}$ & $\begin{array}{l}\text { Risk Ratio } \\
\text { M-H. Fixed, } 95 \% \mathrm{Cl}\end{array}$ \\
\hline Motzer RJ 2013 & 245 & 557 & 282 & 553 & $92.3 \%$ & $0.86[0.76,0.98]$ & \\
\hline Powles T 2012 & 4 & 34 & 11 & 43 & $3.2 \%$ & $0.46[0.16,1.32]$ & \\
\hline Racsa PN 2015 & 10 & 92 & 22 & 199 & $4.5 \%$ & $0.98[0.49,1.99]$ & \\
\hline Total $(95 \% \mathrm{Cl})$ & & 683 & & 795 & $100.0 \%$ & $0.86[0.76,0.97]$ & $\checkmark$ \\
\hline Total events & 259 & & 315 & & & & \\
\hline $\begin{array}{l}\text { Heterogeneity: } \mathrm{Chi}^{2}= \\
\text { Test for overall effect: }\end{array}$ & $\begin{array}{l}.50, \mathrm{df}=2 \\
Z=2.50(\mathrm{~F}\end{array}$ & $\begin{array}{l}2(P=0 \\
P=0.01\end{array}$ & $0.47) ; 1^{2}=$ & & & & 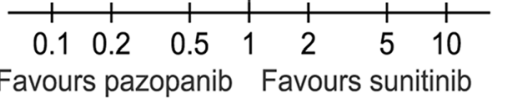 \\
\hline $\begin{array}{l}\text { D } \\
\text { Study or Subgroup }\end{array}$ & $\begin{array}{l}\text { pazopa } \\
\text { Events }\end{array}$ & $\begin{array}{l}\text { nib } \\
\text { Total }\end{array}$ & $\begin{array}{l}\text { sunitir } \\
\text { Events }\end{array}$ & $\begin{array}{l}\text { nib } \\
\text { Total } \\
\end{array}$ & Weight & $\begin{array}{l}\text { Risk Ratio } \\
\text { M-H. Fixed, } 95 \% \mathrm{Cl}\end{array}$ & $\begin{array}{c}\text { Risk Ratio } \\
\text { M-H. Fixed, } 95 \% \mathrm{Cl}\end{array}$ \\
\hline Escudier B 2014 & 12 & 86 & 15 & 82 & $8.0 \%$ & $0.76[0.38,1.53]$ & \\
\hline Kim JH 2016 & 5 & 72 & 14 & 100 & $6.1 \%$ & $0.50[0.19,1.32]$ & \\
\hline Lalani AA 2017 & 41 & 93 & 190 & 577 & $27.6 \%$ & $1.34[1.04,1.73]$ & \\
\hline Motzer RJ 2013 & 134 & 557 & 111 & 553 & $58.3 \%$ & $1.20[0.96,1.50]$ & \\
\hline Total $(95 \% \mathrm{Cl})$ & & 808 & & 1312 & $100.0 \%$ & $1.16[0.98,1.37]$ & 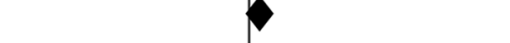 \\
\hline Total events & 192 & & 330 & & & & \\
\hline \multicolumn{5}{|c|}{$\begin{array}{l}\text { Heterogeneity: } \mathrm{Chi}^{2}=5.59, \mathrm{df}=3(P=0.13) ; I^{2}=46 \% \\
\text { Test for overall effect: } Z=1.77(P=0.08)\end{array}$} & & & 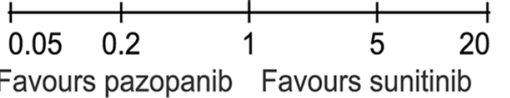 \\
\hline
\end{tabular}

\section{Discussion}

This is the first meta-analysis of the anti-tumor effectiveness, toxicity, and PPPM between pazopanib and sunitinib for treating mRCC or aRCC. Our analysis of 14 medium- to high-quality studies showed the two TKIs had equivalent anti-tumor effectiveness (PFS, OS, ORR, DCR), but sunitinib was associated with more all-grade/ grade 3-4 fatigue, thrombocytopenia, neutropenia and higher PPPM. Additionally, pazopanib had more serious liver toxicity. In subgroup analysis, the pooled outcomes of US studies suggested that pazopanib may have longer OS and higher ORR.

Anti-tumor effectiveness is the most predominant cornerstone to consider when comparing pazopanib and sunitinib. The pooled analysis indicated no significant differences for OS, PFS, ORR, and DCR between 
Table 2 Top 10 adverse effects (all grade) associated with pazopanib versus sunitinib

\begin{tabular}{|c|c|c|c|c|c|c|c|}
\hline \multirow[t]{2}{*}{ Adverse effects } & \multirow{2}{*}{$\begin{array}{l}\text { The } \\
\text { number } \\
\text { of study }\end{array}$} & \multirow{2}{*}{$\begin{array}{l}\text { Pazopanib } \\
\text { group } \\
\text { (event/ } \\
\text { total) }\end{array}$} & \multirow{2}{*}{$\begin{array}{l}\text { Sunitinib } \\
\text { group } \\
\text { (event/ } \\
\text { total) }\end{array}$} & \multirow[t]{2}{*}{ RR $(95 \% \mathrm{Cl})$} & \multirow[t]{2}{*}{$P$ value } & \multicolumn{2}{|c|}{ Heterogeneity } \\
\hline & & & & & & $\overline{P(\%)}$ & $P$ value \\
\hline Diarrhea & 3 & $378 / 714$ & $413 / 1210$ & $1.11[1.01-1.23]$ & 0.03 & 11 & 0.32 \\
\hline Fatigue & 3 & $350 / 714$ & $542 / 1190$ & $0.87[0.79-0.96]$ & 0.006 & 0 & 0.64 \\
\hline Hypertension & 4 & $761 / 1241$ & 783/1747 & $1.04[0.99-1.10]$ & 0.15 & 54 & 0.09 \\
\hline Nausea/Nomiting & 3 & $434 / 719$ & $531 / 1225$ & $0.95[0.88-1.02]$ & 0.15 & 80 & 0.006 \\
\hline Leukopenia & 2 & $258 / 626$ & $477 / 648$ & $0.55[0.50-0.61]$ & $<0.00001$ & 0 & 0.90 \\
\hline Thrombocytopenia & 3 & 259/719 & $529 / 1225$ & $0.54[0.48-0.60]$ & $<0.00001$ & 0 & 0.47 \\
\hline Neutropenia & 3 & $227 / 719$ & $447 / 1225$ & $0.54[0.48-0.61]$ & $<0.00001$ & 0 & 0.57 \\
\hline Increased creatinine & 2 & $190 / 626$ & $284 / 648$ & $0.68[0.59-0.79]$ & $<0.00001$ & 0 & 0.35 \\
\hline Increased AST & 2 & $359 / 626$ & $358 / 648$ & $1.02[0.93-1.12]$ & 0.67 & 0 & 0.96 \\
\hline Increased ALT & 2 & $351 / 626$ & $266 / 648$ & 1.35 [1.20-1.51] & $<0.00001$ & 11 & 0.29 \\
\hline
\end{tabular}

Abbreviations: ALT: alanine aminotransferase, AST: aspartate aminotransferase

pazopanib and sunitinib. A phase III RCT indicated pazopanib had comparable anti-tumor efficacy compared with sunitinib [7]. Furthermore, a retrospective observational study on the experiences of two Turkish hospitals demonstrated that pazopanib and sunitinib were similarly effective for treating mRCC [24]. Similarly, an article with 10-year results from a single-center study found no intergroup differences for treatment effectiveness [25]. Notably, subgroup analysis showed that the US studies had longer OS and higher ORR (Table 4), which suggested that pazopanib might have better anti-tumor effectiveness than sunitinib among American patients with $\mathrm{mRCC}$ or aRCC. The pooled results of Korean studies (95\% CI: $0.49-0.99, P=0.04$ ) also indicated that pazopanib may prolong OS, but the limited number of studies (i.e., one) might weaken the certainty of this result. Additionally, the pooled results of RCT revealed pazopanib may better ORR although the difference wasn't significant. Nevertheless, these conclusions of sub-analysis need be accepted carefully and require further large-sample, well-designed RCTs for confirmation.

The effect of drug toxicity is a significant factor when choosing pazopanib or sunitinib. Here, we observed high rates of drug reduction, diarrhea, fatigue, and hematologic toxicity (leukopenia, thrombocytopenia, neutropenia, increased creatinine) in the sunitinib group. A probable reason might be the inappropriate use of contemporary dose and schedule alterations when using sunitinib. In fact, in a phase-II RCT, Lee et al. found that therapy with $50 \mathrm{mg}$ sunitinib daily using a $2 / 1$ dosing schedule (2 weeks on; 1 week off) offered fewer AEs among mRCC patients than the standard 4/2 schedule (4 weeks on; 2 weeks of) (NCT00570882) after 30 months follow-up [26]. Simalarily, a retrospective analysis showed that therapy with $50 \mathrm{mg}$ sunitinib daily using a $2 / 1$ dosing schedule was associated with better tolerability and less toxicity among

Table 3 Top 10 adverse effects (3-4 grade) associated with pazopanib versus sunitinib

\begin{tabular}{|c|c|c|c|c|c|c|c|}
\hline \multirow[t]{2}{*}{ Adverse effects } & \multirow{2}{*}{$\begin{array}{l}\text { The } \\
\text { number } \\
\text { of study }\end{array}$} & \multirow{2}{*}{$\begin{array}{l}\text { Pazopanib } \\
\text { group } \\
\text { (event/ } \\
\text { total) }\end{array}$} & \multirow{2}{*}{$\begin{array}{l}\text { Sunitinib } \\
\text { group } \\
\text { (event/ } \\
\text { total) }\end{array}$} & \multirow[t]{2}{*}{ RR $(95 \%$ Cl) } & \multirow[t]{2}{*}{$P$ value } & \multicolumn{2}{|c|}{ Heterogeneity } \\
\hline & & & & & & $\bar{P}(\%)$ & $P$ value \\
\hline Diarrhea & 3 & $51 / 660$ & $43 / 691$ & $1.19[0.81-1.74]$ & 0.38 & 0 & 0.42 \\
\hline Fatigue & 3 & $60 / 660$ & $102 / 691$ & $0.59[0.44-0.80]$ & 0.0006 & 0 & 0.49 \\
\hline Hypertension & 2 & $83 / 626$ & $93 / 648$ & $0.43[0.05-3.53]$ & 0.43 & 78 & 0.03 \\
\hline Nausea/Nomiting & 3 & $25 / 660$ & 29/691 & $0.87[0.52-1.46]$ & 0.61 & 0 & 0.61 \\
\hline Leukopenia & 2 & $6 / 626$ & $39 / 648$ & $0.26[0.03-2.55]$ & 0.25 & 84 & 0.01 \\
\hline Thrombocytopenia & 2 & $20 / 626$ & $131 / 648$ & $0.16[0.10-0.25]$ & $<0.00001$ & 0 & 0.37 \\
\hline Neutropenia & 2 & 28/626 & $127 / 648$ & $0.23[0.15-0.34]$ & $<0.00001$ & 0 & 0.97 \\
\hline Increased creatinine & 2 & $4 / 626$ & $8 / 648$ & $0.49[0.15-1.63]$ & 0.25 & NA & NA \\
\hline Increased AST & 2 & $71 / 626$ & $16 / 648$ & $4.46[2.62-7.58]$ & $<0.00001$ & 0 & 0.69 \\
\hline Increased ALT & 2 & $99 / 626$ & $23 / 648$ & $4.34[2.79-6.75]$ & $<0.00001$ & 0 & 0.40 \\
\hline
\end{tabular}




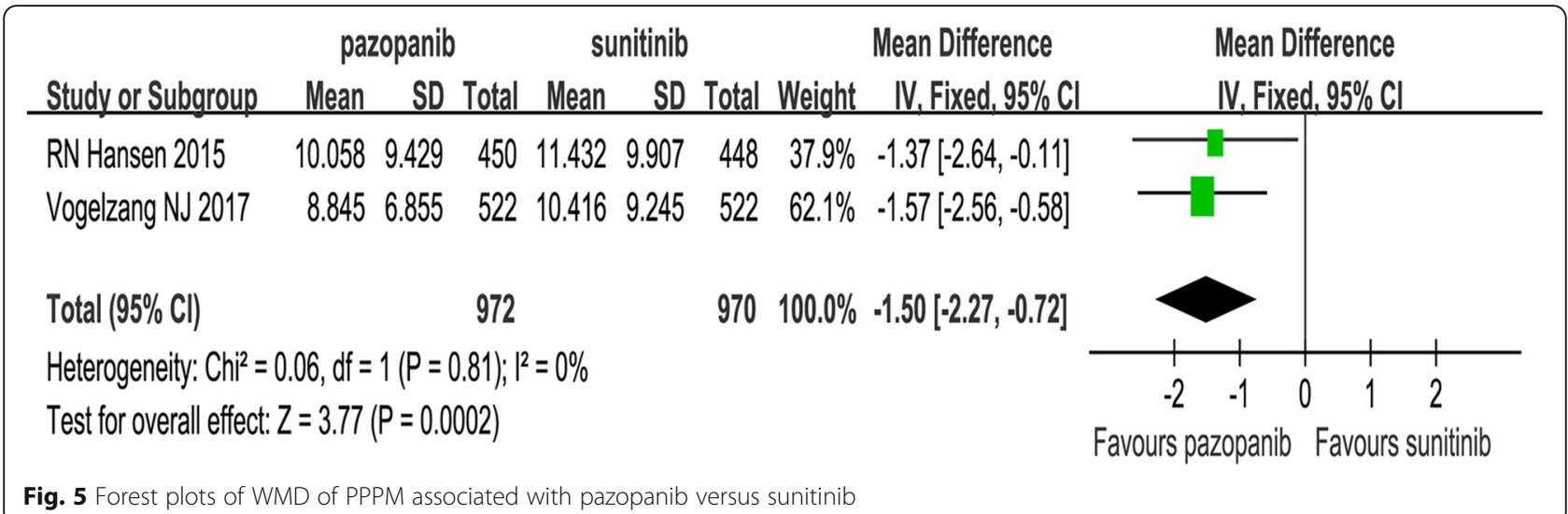

Table 4 Subgroup analysis for progression-free survival, overall survival and objective response rate

\begin{tabular}{|c|c|c|c|c|c|c|c|c|c|c|c|c|}
\hline \multirow[t]{2}{*}{ Group } & \multicolumn{4}{|l|}{ PFS } & \multicolumn{4}{|l|}{ OS } & \multicolumn{4}{|l|}{ ORR } \\
\hline & $\begin{array}{l}\text { No.of } \\
\text { studies }\end{array}$ & HR $(95 \% \mathrm{Cl})$ & $P$ & $P^{2}(\%)$ & $\begin{array}{l}\text { No.of } \\
\text { studies }\end{array}$ & HR (95\% Cl) & $P$ & $P^{2}(\%)$ & No.of studies & RR $(95 \% \mathrm{Cl})$ & $P$ & $P^{2}(\%)$ \\
\hline Total & 3 & $\begin{array}{l}1.06 \\
{[0.98,1.15]}\end{array}$ & 0.13 & 0 & 8 & $\begin{array}{l}0.92 \\
{[0.79-1.07]}\end{array}$ & 0.29 & 61 & 8 & $\begin{array}{l}1.03 \\
{[0.93,1.13]}\end{array}$ & 0.58 & 48 \\
\hline \multicolumn{13}{|l|}{ Nation } \\
\hline USA & 1 & $\begin{array}{l}1.05 \\
{[0.90,1.22]}\end{array}$ & 0.53 & NA & 4 & $\begin{array}{l}0.86 \\
{[0.77,0.95]}\end{array}$ & 0.004 & 28 & 2 & $\begin{array}{l}1.24 \\
{[1.03,1.51]}\end{array}$ & 0.03 & 0 \\
\hline Canada & 1 & $\begin{array}{l}1.08 \\
{[0.98,1.19]}\end{array}$ & 0.12 & NA & 2 & $\begin{array}{l}1.25 \\
{[0.78,1.98]}\end{array}$ & 0.35 & 75 & 1 & $\begin{array}{l}0.91 \\
{[0.81,1.04]}\end{array}$ & 0.16 & NA \\
\hline Korea & 1 & $\begin{array}{l}0.91 \\
{[0.64,1.30]}\end{array}$ & 0.62 & NA & 1 & $\begin{array}{l}0.70 \\
{[0.49,0.99]}\end{array}$ & 0.04 & NA & 1 & $\begin{array}{l}1.57 \\
{[0.98,2.52]}\end{array}$ & 0.06 & NA \\
\hline Italy & NA & NA & NA & NA & 1 & $\begin{array}{l}0.94 \\
{[0.38,2.32]}\end{array}$ & 0.89 & NA & 2 & $\begin{array}{l}1.28 \\
{[0.90,1.82]}\end{array}$ & 0.17 & NA \\
\hline UK & NA & NA & NA & NA & NA & NA & NA & NA & 1 & $\begin{array}{l}1.05 \\
{[0.35,3.16]}\end{array}$ & 0.93 & NA \\
\hline France & NA & NA & NA & NA & NA & NA & NA & NA & 1 & $\begin{array}{l}0.89 \\
{[0.48,1.63]}\end{array}$ & 0.70 & NA \\
\hline \multicolumn{13}{|l|}{$\begin{array}{l}\text { The number of } \\
\text { pazopanib }\end{array}$} \\
\hline$>100$ & 2 & $\begin{array}{l}1.07 \\
{[0.99,1.16]}\end{array}$ & 0.1 & 0 & 3 & $\begin{array}{l}0.93 \\
{[0.81,1.06]}\end{array}$ & 0.26 & 60 & 2 & $\begin{array}{l}1.05 \\
{[0.78,1.42]}\end{array}$ & 0.73 & 85 \\
\hline$<100$ & 1 & $\begin{array}{l}0.91 \\
{[0.64,1.30]}\end{array}$ & 0.62 & NA & 5 & $\begin{array}{l}0.95 \\
{[0.65,1.38]}\end{array}$ & 0.77 & 69 & 6 & $\begin{array}{l}1.25 \\
{[0.96,1.62]}\end{array}$ & 0.09 & 0 \\
\hline \multicolumn{13}{|l|}{ classification a } \\
\hline Poor risk & 1 & $\begin{array}{l}0.91 \\
{[0.64,1.30]}\end{array}$ & 0.62 & NA & 2 & $\begin{array}{l}0.90 \\
{[0.56,1.44]}\end{array}$ & 0.66 & 78 & 1 & $\begin{array}{l}1.57 \\
{[0.98,2.52]}\end{array}$ & 0.06 & NA \\
\hline Intermediate risk & NA & NA & NA & NA & 2 & $\begin{array}{l}1.36 \\
{[0.73,2.52]}\end{array}$ & 0.33 & 73 & NA & NA & NA & NA \\
\hline Mixed group & 2 & $\begin{array}{l}1.07 \\
{[0.99,1.16]}\end{array}$ & 0.1 & 0 & 7 & $\begin{array}{l}0.95 \\
{[0.82,1.11]}\end{array}$ & 0.54 & 61 & 7 & $\begin{array}{l}1.01 \\
{[0.91,1.11]}\end{array}$ & 0.85 & 43 \\
\hline \multicolumn{13}{|l|}{ Study design } \\
\hline RS & 2 & $\begin{array}{l}1.07 \\
{[0.97,1.17]}\end{array}$ & 0.17 & 0 & 7 & $\begin{array}{l}0.93 \\
{[0.77,1.12]}\end{array}$ & 0.44 & 67 & 5 & $\begin{array}{l}1.17 \\
{[0.85,1.61]}\end{array}$ & 0.33 & 58 \\
\hline $\mathrm{RCT}$ & 1 & $\begin{array}{l}1.05 \\
{[0.90,1.22]}\end{array}$ & 0.53 & NA & 1 & $\begin{array}{l}0.91 \\
{[0.76,1.08]}\end{array}$ & 0.29 & NA & 3 & $\begin{array}{l}1.19 \\
{[1.00,1.43]}\end{array}$ & 0.05 & 0 \\
\hline
\end{tabular}

Abbreviations: PFS: progression-free survival, OS: overall survival, ORR: objective response rate, HR, hazard ratio, RR: relative risk, RS: retrospective study, RCT: randomized controlled trial, NA: not available

a Patients were classified according to the International mRCC Database Consortium (IMDC) risk group 
patients with mRCC as compared with the standard 4/2 schedule [27]. Although their survival time might not have been decreased, sunitinib-treated patients had slightly fewer clinical benefits based on quality-adjusted time without symptoms or toxicity (Q-TWiST) scores, which were used for evaluating the survival quality of patients, especially when the quality of life associated with tumor progression was higher than the quality of life related to AEs [28, 29]. In fact, this difference may be of vital importance for patients, as treatment was administered continuously over months [8]. However, liver toxicity (grade 3-4) was more frequent in the pazopanib group [7]. It was necessary to use hepatic protection drugs and monitor liver function periodically in patients using pazopanib, which may have helped prevent more severe hepatotoxic AEs. Similarly, periodic hematological examination and other relevant tests for sunitinib-treated patients were also significant. Moreover, in PISCES, a randomized, double-blind, crossover clinical trial comparing the influences of the two drugs' toxicity and tolerability on the preferences of patients diagnosed with mRCC, 70\% of patients and $61 \%$ of physicians preferred pazopanib over sunitinib [21]. In the COMPARZ trial, pazopanib-treated patients had superior health-related quality-of-life scores for both primary end points (fatigue and treatment AEs) during the first 6 months of therapy compared with sunitinib-treated patients [30]. In fact, physicians needed to be exceedingly cautious in administering pazopanib to patients with poor liver function.

The effect of total costs is also an indispensable factor when choosing between the two TKIs. Our results also proved that pazopanib had a significantly lower PPPM than sunitinib (Fig. 5). Delea et al. suggested that pazopanib was more cost-effective compared with sunitinib when used as first-line treatment among American patients with mRCC [31]. Other studies have reported similar results [18, 32-35]. Although the two TKIs did not have greatly different costs, the lower costs of pazopanib therapy might help relieve the financial burden on patients and their family, extend treatment time, and even relieve the psychological pressure on patients facing with so high expenditure, especially patients from impoverished families and from the developing countries.

The sensitivity analysis was performed to analyze the moderate-significant heterogeneity (I2 $>50 \%$ ) of OS, ORR and DCR. We assumed that Lalani AA et al. [9] in OS, Ruiz-Morales et al. [18] in ORR, and Kim JH et al. [13] in DCR might be the drivers of the moderatesignificant heterogeneity. In the study Lalani AA et al. [9], there were less patients with Karnofsky performance score $<80$ in the sunitinib group than the pazopanib group ( $17 \%$ vs. $26 \%)$, and they would have longer survival. As a result, sunitinib-treated patients of the study Lalani AA et al. [9] had better OS. In addition, the sensitivity analysis of ORR showed that this result was not very robust, and the estimate of the study Ruiz-Morales et al. [18] exceeded the 95\% CI. We found that patients in the sunitinib group were younger than pazopanib-treated patients (median age: 62 vs. $65, P$ $<0.0001$ ) in the study of Ruiz-Morales et al. [18], which might cause better ORR. In a retrospective study, Hutson et al. reported more treatment-emergent AEs were recorded in older patients using sunitinib [36]. Older patients might have more drug discontinuation or reductions, which inevitably influenced response rate of targeted drugs. Therefore, sunitinib-treated patients of the study Ruiz-Morales et al. [18] had better ORR. Additionally, we found that there were all poor-risk patients with $\mathrm{mRCC}$ in the study $\mathrm{Kim} \mathrm{JH}$ et al. [13], which might give rise to significantly poor DCR of sunitinib-treated patients.

Several limitations should be considered when considering our results. First, the limited number of RCTs (only three) weakened the quality of these outcomes and the inclusion of retrospective studies would exert a certain impact on the reliability of these outcomes. Admittedly, it might increase the heterogeneity when we analyzed PFS and OS if we combined different types of studies. But we had performed the subgroup analysis of PFS and OS about study design in our meta-analysis, and no difference was found in this subgroup analysis between the two groups in terms of PFS and OS. Therefore, the bias caused by the combination of the two different types of studies might be relatively small. Second, there was moderate-significant heterogeneity for some comparisons (OS, ORR and DCR), which weakened the reliability of these outcomes. Third, the limited number of studies on PFS, PPPM, and the subgroup of OS in Korea might have resulted in relatively unreliable estimates. Fourth, the included articles were limited to literature sources published in English, so it might lead to the language bias. However, as a comprehensive and universal language, English studies have more reliable information, so we can guarantee the quality of all included studies if the only English studies were included. Fifth, we could not control completely for confounding factors (pre-treatment, pathological type), which were unavailable for some studies but which could influence final results. Sixth, some specific subsequent costs were part of PPPM, but they didn't have direct association with the anti-tumor effectiveness. Accordingly, we suggested that total healthcare costs should be elaborated if possible in future drug evaluation studies.

Our meta-analysis showed that pazopanib had similar anti-tumor effectiveness for mRCC or aRCC as compared with sunitinib. But pazopanib may be more suitable to poor patients due to lower PPPM. Great care should be taken when using pazopanib to treat patients 
with abnormal liver function. In addition, pazopanib might have more benifits (better OS, higher ORR) among American patients with mRCC or aRCC. Nevertheless, the inherent limitations of this meta-analysis mean that additional large-scale, high-quality studies are required to better determine the role of the two targeted drugs under complicated clinical circumstances.

\section{Additional files}

Additional file 1: Table S1. PRISMA 2009 Checklist. (DOC 65 kb)

Additional file 2: Figure S1. Sensitivity analysis of PFS (A) and OS (B) (TIF $22670 \mathrm{~kb}$ )

Additional file 3: Figure S2. Sensitivity analysis of ORR (A) and DCR (B) (TIF $1268 \mathrm{~kb}$ )

Additional file 4: Figure S3. Begg's and Egger's tests for comparisons of HR of PFS (A) and OS (B) associated with pazopanib versus sunitinib. (TIF $8671 \mathrm{~kb}$ )

Additional file 5: Figure S4. Begg's and Egger's tests for comparisons of ORR (A) and DCR (B) associated with pazopanib versus sunitinib. (TIF $1210 \mathrm{~kb}$ )

\section{Abbreviations}

AEs: Adverse effects; ALT: Alanine aminotransferase; aRCC: Advanced renal cell carcinoma; AST: Aspartate aminotransferase; Cls: Confidence intervals; DCR: Disease control rate; HRs: Hazard ratios; mRCC: Metastatic renal cell carcinoma; ORR: Objective response rate; OS: Overall survival; PFS: Progression-free survival; PPPM: Per-patient-per-month costs; RCT: Randomized controlled trial; RRs: Risk ratios; TKl: Tyrosine kinase inhibitor; WMD: Weighted mean difference

\section{Acknowledgements}

The authors thank professor Jichun Liu, MD (Department of Cardio-Thoracic Surgery, The second affiliated hospital of Nanchang University) for his advice and professor Xiaoshu Cheng, MD, PhD (Department of Cardiology, The second affiliated hospital of Nanchang University) for his data collection.

\section{Availability of data and material}

All data is available in this paper.

\section{Funding}

This study was supported by National Natural Science Foundation of China (NSFC), with no commercial entity involved, number of grants (81560345). Role of the Funding: The NSFC had no role in the design and conduct of the study; collection, management, analysis, and interpretation of the data; preparation, review, or approval of the manuscript; and decision to submit the manuscript for publication.

\section{Authors' contributions}

HD conceived of the idea, designed the study, interpreted the data and wrote the manuscript. YH interpreted the data and performed the study through STATA. ZDH interpreted the data and other relevant information. XHY and ZC interpreted the data and other relevant information. YPW provided the examination for the methodology, reviewed and revised our manuscript. WXZ conceived of the idea, designed the study, searched the relevant database, wrote the manuscript and confirmed statistical analysis. All authors read and approved the final manuscript.

\section{Ethics approval and consent to participate}

Not applicable.

\section{Consent for publication}

Not applicable.

\section{Competing interests}

The authors declare that they have no competing interests.

\section{Publisher's Note}

Springer Nature remains neutral with regard to jurisdictional claims in published maps and institutional affiliations.

\section{Author details}

'Department of Thoracic Surgery, The First Affiliated Hospital of Nanchang

University, Minde Rd, Nanchang 330006, Jiangxi Province, China. ${ }^{2}$ Jiangxi

Medical College, Nanchang University, Nanchang 330006, China.

${ }^{3}$ Department of Urology, The Second Affiliated Hospital of Nanchang

University, Nanchang 330006, China.

Received: 29 November 2018 Accepted: 10 May 2019

Published online: 23 May 2019

\section{References}

1. Siegel RL, Miller KD, Jemal A, et al. Cancer statistics, 2018. CA Cancer J Clin. 2018;68(1):7-30.

2. Janzen NK, Kim HL, Figlin RA, et al. Surveillance after radical or partial nephrectomy for localized renal cell carcinoma and management of recurrent disease. Urol Clin North Am. 2003;30:843-52.

3. Escudier B, Eisen T, Porta C, et al. Renal cell carcinoma: ESMO clinical practice guidelines for diagnosis, treatment and follow-up. Ann Oncol. 2012; 23(Suppl 7):vii65-71.

4. Motzer RJ, Agarwal N, Beard C, et al. NCCN clinical practice guidelines in oncology: kidney cancer. J Natl Compr Cancer Netw. 2009; 7:618-30.

5. Gore ME, Szczylik C, Porta C, et al. Safety and efficacy of Sunitinib for metastatic renal-cell carcinoma: an expanded-access trial. Lancet Oncol. 2009;10(8):757-63.

6. Sternberg CN, Davis ID, Mardiak J, et al. Pazopanib in locally advanced or metastatic renal cell carcinoma: results of a randomized phase III trial. J Clin Oncol. 2010;28(6):1061-8.

7. Motzer RJ, Hutson TE, Cella D, et al. Pazopanib versus sunitinib in metastatic renal-cell carcinoma. N Engl J Med. 2013;369(8):722-31.

8. Powles $T$, Sarwar $N$, Jones $R$, et al. An indirect comparison of the toxicity of sunitinib and pazopanib in metastatic clear cell renal cancer. Eur J Cancer. 2012;48(17):3171-6.

9. Lalani AA, Li H, Heng DYC, et al. First-line sunitinib or pazopanib in metastatic renal cell carcinoma: The Canadian experience. Can Urol Assoc J. 2017;11(3-4):112-7.

10. Tierney JF, Stewart LA, Ghersi D, Burdett S, Sydes MR. Practical methods for incorporating summary time-to-event data into meta-analysis. Trials. 2007:8:16.

11. Jadad AR, Moore RA, Carroll D, et al. Assessing the quality of reports of randomized clinical trials: is blinding necessary? Control Clin Trials. 1996;17(1):1-12.

12. Wells GA, Shea BJ, O'Connell D, et al. The Newcastle-Ottawa scale (nos) for assessing the quality of non-randomized studies in meta-analysis. Appl Eng Agric. 2014;18(6):727-34

13. Kim JH, Park I, Lee $J$, et al. Pazopanib versus sunitinib for the treatment of metastatic renal cell carcinoma patients with poor-risk features. Cancer Chemother Pharmacol. 2016;78(2):325-32.

14. Byfield SA, McPheeters JT, Burton TM, et al. Persistence and compliance among U.S. patients receiving Pazopanib or Sunitinib as first-line therapy for advanced renal cell carcinoma: a retrospective claims analysis. J Manag Care Spec Pharm. 2015;21(6):515-22.

15. Pal SK, Ghate SR, Li N, et al. Real-World Survival Outcomes and Prognostic Factors among patients receiving first targeted therapy for advanced renal cell carcinoma: a SEER-Medicare database analysis. Clin Genitourin Cancer. 2017;15(4):e573-82.

16. Santoni M, Conti A, Porta C, et al. Sunitinib, Pazopanib or Sorafenib for the treatment of patients with late relapsing metastatic renal cell carcinoma. J Urol. 2015;193(1):41-7.

17. Vogelzang NJ, Pal SK, Ghate SR, et al. Clinical and economic outcomes in elderly advanced renal cell carcinoma patients starting Pazopanib or Sunitinib treatment: a retrospective Medicare claims analysis. Adv Ther. 2017:34(11):2452-65.

18. Hansen RN, Hackshaw MD, Nagar SP, et al. Health care costs among renal Cancer patients using Pazopanib and Sunitinib. J Manag Care Spec Pharm. 2015;21(1):37-44 44a-d. 
19. Racsa PN, Whisman TR, Worley K, et al. Comparing two tyrosine kinase inhibitors for treatment of advanced renal cell carcinoma in Medicare and commercially insured patients. Curr Med Res Opin. 2015;31(10):1933-40.

20. Bianconi M, Faloppi L, Loretelli C, et al. Angiogenesis genotyping in the selection of first-line treatment with either sunitinib or pazopanib for advanced renal cell carcinoma. Oncotarget. 2016;7(25):37599-607.

21. Escudier B, Porta C, Bono P, et al. Randomized, controlled, double-blind, cross-over trial assessing treatment preference for Pazopanib versus Sunitinib in patients with metastatic renal cell carcinoma: PISCES study. J Clin Oncol. 2014;32(14):1412-8.

22. Ruiz-Morales JM, Swierkowski M, Wells JC, et al. First-line sunitinib versus pazopanib in metastatic renal cell carcinoma: results from the international metastatic renal cell carcinoma database consortium. Eur J Cancer. 2016;65:102-8.

23. Kucharczyk J, Mandalapu K, Satti S, et al. Outcomes of patients with laterelapse metastatic renal cell carcinoma treated with targeted therapies: a single institution experience. Ochsner J. 2017;17(4):331-4.

24. Ekenel M, Aydin E, Cil I, et al. Sunitinib versus pazopanib for patients with metastatic renal cell carcinoma: two Turkish hospital experience, a retrospective comparative case series study. Ann Oncol. 2017; 28(suppl_5):367.

25. Chow S, Pillai MR, Galvis V, et al. 10 year outcomes from a single-Centre experience of sunitinib and pazopanib as first-line treatment of metastatic renal-cell carcinoma (mRCC). J Clin Oncol. 2016;34(15):e16085.

26. Lee JL, Kim MK, Park I, et al. RandomizEd phase II trial of Sunitinib four weeks on and two weeks off versus two weeks on and one week off in metastatic clear-cell type REnal cell carcinoma: RESTORE trial. Ann Oncol. 2015;26(11):2300-5.

27. Najjar YG, Mittal K, Elson P, et al. A 2 weeks on and 1 week off schedule of sunitinib is associated with decreased toxicity in metastatic renal cell carcinoma. Eur J Cancer. 2014;50(6):1084-9.

28. Beaumont JL, Salsman JM, Diaz J, et al. Quality-adjusted time without symptoms or toxicity analysis of pazopanib versus sunitinib in patients with renal cell carcinoma. Cancer. 2016;122(7):1108-15.

29. Granovetter M. Benefits of pazopanib over sunitinib for renal cell carcinoma Lancet Oncol. 2016;17(3):e93.

30. Hackshaw MD, Diaz J, Huang C, et al. Quality of life (QoL) among patients with renal cell carcinoma (RCC) treated with pazopanib versus sunitinib in the COMPARZ study. J Clin Oncol. 2013;31(6_suppl:346.

31. Delea TE, Amdahl J, Diaz J, et al. Cost-effectiveness of pazopanib versus sunitinib for renal cancer in the United States. J Manag Care Spec Pharm. 2015;21(1):46-54.

32. Liviu Preda A, Galieta Mincă D. Cost-Effectiveness Analysis of Treatment for Metastatic Renal Carcinoma in Romania. J Med Life. 2018;11(4):306-11.

33. Capri S, Porta C, Delea TE, et al. Cost-effectiveness of Pazopanib versus Sunitinib as first-line treatment for locally advanced or metastatic renal cell carcinoma from an Italian National Health Service Perspective. Clin Ther. 2017:39(3):567-80

34. Amdahl J, Diaz J, Sharma A, et al. Cost-effectiveness of pazopanib versus sunitinib for metastatic renal cell carcinoma in the United Kingdom. PLoS One. 2017;12(6):e0175920.

35. Anaya $P$, Delea TE, Pichardo $P$, et al. Cost effectiveness analysis based on progression free survival (PFS) of Pazopanib versus Sunitinib for the treatment of advanced renal cell carcinoma (ARCC) in the Mexican context. Value Health. 2012;15(4):A220

36. Hutson TE, Bukowski RM, Rini Bl, et al. Efficacy and safety of sunitinib in elderly patients with metastatic renal cell carcinoma. $\mathrm{Br} J$ Cancer. 2014;110(5):1125-32.

\section{Ready to submit your research? Choose BMC and benefit from:}

- fast, convenient online submission

- thorough peer review by experienced researchers in your field

- rapid publication on acceptance

- support for research data, including large and complex data types

- gold Open Access which fosters wider collaboration and increased citations

- maximum visibility for your research: over $100 \mathrm{M}$ website views per year

At BMC, research is always in progress.

Learn more biomedcentral.com/submissions 